\title{
An evaluation of community-based cognitive stimulation therapy: a pilot study with an Irish population of people with dementia
}

\author{
M. E. Kelly'*, S. Finan, M. Lawless, N. Scully, J. Fitzpatrick, M. Quigley, F. Tyrrell, A. O’Regan and A. Devane \\ Department of Psychology, National University of Ireland Maynooth, Maynooth, Co. Kildare, Ireland
}

\begin{abstract}
Objectives. Research shows that cognitive stimulation therapy (CST) improves cognitive function, quality of life, and well-being of people with mild-moderate dementia. Despite consistent evidence and recommendations, CST is not routinely available in Ireland post-diagnosis. The aim of the current research was to develop and evaluate communitybased CST for people with mild-moderate dementia, run by the Alzheimer Society of Ireland across four pilot sites in Ireland.
\end{abstract}

\begin{abstract}
Methods. Participants with mild-moderate dementia attended once weekly CST sessions for 14 weeks. Baseline and post-intervention assessments were completed by CST participants, carers, and CST facilitators. Primary outcomes of interest for CST participants included quality of life (Quality of Life in Alzheimer Disease Scale), cognitive function (Montreal Cognitive Assessment), and subjective cognitive function (Memory Awareness Rating Scale-Functioning Subscale). Secondary outcomes included well-being, cognitive ability, satisfaction with cognitive performance, and engagement and confidence of CST participants; well-being of carers; and job satisfaction of facilitators. Post-intervention interviews supplemented quantitative analyses.

Results. In total, 20 CST participants, 17 carers, and six CST facilitators completed evaluation assessments. Results showed that CST improved participants' satisfaction with cognitive performance $(p=0.002)$, level of engagement $(p=0.046)$, level of confidence $(p=0.026)$. Improvements on subjective cognitive function just fell short of significance $(p=0.055)$. Qualitative analysis of interview data identified consistent themes of cognitive and overall benefits of CST; and provided support for quantitative data.

Conclusions. Community-based CST positively impacted the lives of people with dementia and their families. This study supports prior recommendations that CST should be made routinely available to people with mild-moderate dementia, particularly in light of the lack of post-diagnostic interventions currently offered in Ireland.
\end{abstract}

Received 25 January 2016; Revised 14 March 2016; Accepted 24 May 2016; First published online 14 July 2016

Key words: Cognitive stimulation, dementia, early intervention, post-diagnosis.

\section{Introduction}

Cognitive stimulation therapy (CST) is a nonpharmacological intervention for people with mild-moderate dementia that provides stimulation for thinking, concentration, and memory, typically conducted in a small group setting (Woods et al. 2012). Cognitive interventions may offer benefits to people with dementia early in the disease course in terms of maintaining existing cognitive function, delaying further decline, and improving subjective outcomes (Clare, 2008; Batsch \& Miller, 2009). There is growing evidence that the brain can recruit additional neural networks in early stage dementia (Grady et al. 2003), and that new learning can be achieved (Clare, 2008).

* Address for correspondence: M. E. Kelly, Department of Psychology, National University of Ireland Maynooth, Room SF21, 2nd Floor John Hume, North Campus, Maynooth, Co. Kildare, Ireland.

(Email: Michelle.E.Kelly@nuim.ie)
Cognitive interventions should therefore be a primary focus of service provision for individuals with mild-moderate dementia (Clare et al. 2003).

Several research studies have examined the impact of CST on people with dementia (Spector et al. 2001; Spector et al. 2003; Onder et al. 2005); including one study with an Irish population in long-term care (Coen et al. 2011). Reported outcomes included improvements in cognitive function (Spector et al. 2003; Onder et al. 2005; Coen et al. 2011; Aguirre et al. 2012) and quality of life (Spector et al. 2003; Coen et al. 2011; Aguirre et al. 2012); and reduced anxiety or depression (Spector et al. 2001). More recently, Woods et al. (2012) evaluated 15 randomised control trials (RCTs) of CST, and concluded that CST consistently improved cognitive function, self-reported quality of life, and well-being for people with dementia; and improved staff ratings of clients' communication and social interaction. The positive effects of the intervention remained evident up 
to 3 months after treatment ceased, and benefits were reported to be over and above medication effects (Woods et al. 2012; Aguirre et al. 2013). With the addition of maintenance CST, positive effects could be prolonged even further (Streater et al. 2012; Orrell et al. 2014).

CST is typically evaluated as a 7 week, 14 session programme. Due to time constraints and resource limitations, however, many National Health Service (NHS) services in the United Kingdom deliver the programme once per week over 14 weeks (Cove et al. 2014). One RCT evaluated this method of delivery but reported no significant differences between intervention and control groups on measures of cognitive function and quality of life; suggesting a possible 'dose' requirement of twice weekly sessions (Cove et al. 2014). The authors concluded that additional data were required to establish whether once weekly CST sessions may benefit people with dementia.

\section{User perspectives}

CST is one of the few non-pharmacological interventions with which the views of people with dementia have been taken into account (Pierce, 2012). Spector et al. (2011) conducted a qualitative study in the United Kingdom to investigate if improvements associated with CST were reported by people with dementia, their carers, and CST facilitators. The overall experience of attending CST was reported by both participants and carers as being emotionally positive, and most participants reported improvements in confidence and cognitive benefits.

\section{Cost-effectiveness}

A cost-effectiveness analysis of CST was conducted by Knapp et al. in conjunction with the London School of Economics. Results showed that CST was more costeffective than usual care when considering benefits in cognition and quality of life. There was also evidence that CST might be more cost-effective than dementia medication alone (Knapp et al. 2005). In 2011, the NHS Institute for Innovation and Improvement reported that CST could save the NHS over $£ 54.9$ million/year compared with the use of antipsychotic medication (Matrix Evidence, 2011). Figures presented by Cahill et al. (2012) show that in Ireland, the cost of providing antidementia and anti-anxiety/depression medication is $€ 13$ million and €518000/annum, respectively. As has been shown in the United Kingdom, providing non-pharmacological interventions may significantly reduce these costs. In addition, if the provision of CST reduced the number of people with dementia residing in long-stay care in Ireland by only $10 \%$, this could offer a saving of over $€ 73$ million/annum (based on figures presented by Cahill et al. 2012).

\section{Recommendations for CST}

CST is the only non-pharmacological intervention currently recommended by government guidelines (Spector et al. 2011). The Social Care Institute for Excellence (SCIE) and the National Institute for Health and Clinical Excellence (NICE) published National Clinical Practice Guidelines on supporting people with dementia, and concluded that 'people with mildmoderate dementia of all types should be given the opportunity to participate in a structured group cognitive stimulation programme' (NICE-SCIE, 2007: 25). This recommendation was reiterated by the World Alzheimer Report in 2011 (Prince et al. 2011). In Ireland, in the Health Information and Quality Authority's (HIQA) (2008) report on National Quality Standards promoted the use of cognitive stimulation techniques in dementia-specific care (HIQA, 2008).

\section{An Irish perspective}

Despite consistent recommendations and supporting research evidence, cognitive stimulation interventions are not routinely available to people with dementia in Ireland post-diagnosis. Cahill et al. (2012) identified a 'treatment gap' where early diagnosis is not followedup with early intervention. This absence of focus on post-diagnostic interventions has been reiterated by Irish people with dementia, who have called for improved post-diagnostic services (National Dementia Summit Report, 2012). In 2014, the Irish Government published the National Dementia Strategy (NDS) in which they recognised timely diagnosis and intervention; and integrated supports and services for people with dementia, as two of six 'priority action areas' (Department of Health, 2014). Unfortunately, little has been done to address these priorities since the publication of the NDS. These findings not only identify the palpable lack of availability of evidence-based cognitive stimulation interventions for people with early stage dementia, but also highlight the necessity for the provision of same.

\section{The current research}

The aim of the current research was to evaluate the impact of community-based CST delivered once per week for 14 weeks, on outcomes including quality of life, cognitive function, and subjective cognitive function of people with mild-moderate dementia; and on the well-being of carers. This is the first study of its kind in Ireland. The CST programme was delivered by Alzheimer Society of Ireland (ASI) staff in four pilot 
sites and was evaluated using both qualitative and quantitative research methods.

\section{Method}

\section{Participants}

A total of 28 participants with dementia initially took part in the CST intervention, and 20 completed the study $(n=20)$. Participants were 11 females and nine males, aged between 53 and $86(\mathrm{M}=73$, s.D. $=7.61)$ with a formal diagnosis of dementia. Participants were required to be aware of their diagnosis, be able to give informed consent, and have sufficient communication and comprehension skills to participate in the intervention. Where possible, either the Montreal Cognitive Assessment (MoCA; Nasreddine et al. 2005) or the Mini Mental Status Exam (MMSE; Folstein et al. 1975) was administered by the participants' General Practitioner (GP) before entry into the study. MoCA scores $(n=9)$ ranged from 12 to 25 , with a mean score of 18.22 (S.D. $=4.35)$; whereas MMSE scores $(n=5)$ ranged from 15 to 25 , with a mean score of 20 (s.D. $=4.27$ ). Scores of between 10 and 26 on the MoCA, or 11 and 25 on the MMSE are the suggested cut-offs for mildmoderate levels of cognitive impairment (Nasreddine et al. 2005; Perneczky et al. 2006). Scores for the remaining participants $(n=6)$ were not provided, GPs did, however, clear participants for entry to the study.

Carers completed baseline $(n=20)$ and postintervention $(n=17)$ questionnaires but did not participate in the CST intervention. CST facilitators $(n=8)$ were ASI employees with formal dementia and CST training, and also completed baseline and post-intervention questionnaires $(n=6)$. All facilitators followed intervention guidelines stipulated in CST Manuals; Making a Difference 1 (Spector et al. 2006) and Making a Difference 2 (Aguirre et al. 2011).

\section{Procedure}

Participants were recruited through the ASI. Interested individuals contacted CST facilitators at the relevant sites for information about the intervention and requirements for participation. The study was conceptualised as a repeated measures (pre-post intervention) design; and consisted of 16 sessions in total. Participants completed baseline and post-intervention assessments in sessions one and 16, respectively. The CST intervention was delivered across 14 sessions; from session two to 15 . Where possible, participants attended their GP before and after study sessions to have baseline and post-intervention MoCA assessments conducted. Although the MoCA was predominantly utilised, four baseline MMSE scores were alternatively provided. To allow for comparison, these MMSE scores were converted to MoCA scores using the van Steenoven et al. (2014) score conversion guidelines.

\section{Outcomes}

Primary outcomes of interest were quality of life, cognitive function, and subjective cognitive function of CST participants (self and informant ratings). Secondary outcomes were self-rated cognitive ability, satisfaction with cognitive performance (self and informant ratings), well-being (self and informant ratings), and level of engagement and confidence (facilitator and carer informant ratings) of participants; well-being of carers; and job satisfaction for CST facilitators.

\section{Baseline assessments}

Baseline data were collected during the first session at each site (see Table 1). CST participants completed five baseline measures. Quality of life was measured using the Quality of Life in Alzheimer Disease Scale (QoL-AD; Logsdon et al. 1999), self-rating; cognitive function was assessed with the MoCA (or MMSE); and subjective cognitive functioning was measured using the Memory Awareness Rating Scale-Functioning Subscale (MARS-F; Clare et al. 2002; range 0-52), self-rating. The remaining outcomes of cognitive ability, satisfaction with cognitive performance, and well-being were measured using self-ratings on Likert scales. Each Likert was scored on scale of 1-10, with one being the lowest score possible and ten being the highest score possible.

Carers completed one self-rating baseline measure, and six informant measures. Well-being (self-rated) was measured on a Likert scale (as above). Carer ratings of their loved one's quality of life and subjective cognitive functioning were assessed using standardised measures (QoL-AD and MARS-F). Informant ratings of participants' cognitive ability, levels of engagement and confidence, well-being, and satisfaction with loved one's cognitive performance were measured on Likert scales. A memory functioning discrepancy (MFD)-corrected score was calculated to compare self- and informant ratings on the MARS-F (Clare et al. 2010). Corrected MFD scores close to 0 indicate good agreement; positive scores indicate that participants rate their memory functioning as more positively than their informants, whereas negative scores indicate that participants rate their memory less positively than their informants.

CST facilitators completed one self-rating baseline measures, and two informant measure about CST participants. Level of job satisfaction was rated on a brief job satisfaction survey that included six statements (e.g. 'my work gives me a sense of accomplishment') with the response options $1=$ 'strongly agree' to $5=$ 'strongly 
Table 1. List of outcome measures with mean (S.D.) scores at baseline and post-intervention for cognitive stimulation therapy (CST) participants, carers, and CST facilitators

\begin{tabular}{|c|c|c|c|}
\hline Outcome & Outcome measure & Baseline [M (s.D.)] & Post-CST [M (S.D.)] \\
\hline \multicolumn{4}{|l|}{ CST participants $(n=20)$} \\
\hline \multirow[t]{2}{*}{ Quality of life } & QoL-AD self-rating & $35.25(7.89)$ & $35.80(5.78)$ \\
\hline & QoL-AD informant & $32.65(6.49)$ & $32.40(6.05)$ \\
\hline Cognitive function & $\operatorname{MoCA}(n=14)$ & $17.00(4.86)$ & $17.35(4.36)$ \\
\hline \multirow[t]{3}{*}{ Subjective cognitive function } & MARS-F self-rating* & $28.15(11.17)$ & $34.65(9.21)$ \\
\hline & MARS-F informant & $24.06(9.89)$ & $24.20(9.46)$ \\
\hline & MARS-F MFD & $0.11(0.68)$ & $0.30(0.57)$ \\
\hline \multirow[t]{2}{*}{ Cognitive ability } & Likert - self-rating & $5.45(2.06)$ & $6.25(1.83)$ \\
\hline & Likert - informant & $4.94(2.16)$ & $4.97(1.86)$ \\
\hline \multirow[t]{2}{*}{ Satisfaction with cognitive performance } & Likert - self-rating* & $5.32(1.81)$ & $6.70(1.87)$ \\
\hline & Likert - informant & $4.64(2.23)$ & $5.47(1.91)$ \\
\hline \multirow[t]{2}{*}{ Well-being } & Likert - self-rating & $6.75(2.05)$ & $7.75(1.51)$ \\
\hline & Likert - informant & $5.71(1.57)$ & $6.68(1.93)$ \\
\hline \multirow[t]{2}{*}{ Overall engagement } & Likert - informant-carer & $6.29(2.14)$ & $6.85(1.80)$ \\
\hline & Likert - informant-facilitator* & $6.21(2.47)$ & $7.69(1.67)$ \\
\hline \multirow[t]{2}{*}{ Overall confidence } & Likert - informant-carer & $5.76(1.82)$ & $5.97(2.27)$ \\
\hline & Likert - informant-facilitator* & $5.62(2.5)$ & $7.63(1.93)$ \\
\hline Value of CST & Likert - self-rating & - & $8.33(2.22)$ \\
\hline Satisfaction with CST & Likert - self-rating & - & $8.77(1.59)$ \\
\hline \multicolumn{4}{|l|}{ Carers $(n=17)$} \\
\hline Well-being & Likert - self-rating & $7.27(1.80)$ & $8.15(0.96)$ \\
\hline Value of CST & Likert scale & - & $8.71(2.14)$ \\
\hline Satisfaction with CST & Likert scale & - & $8.94(2.08)$ \\
\hline \multicolumn{4}{|l|}{ CST facilitators $(n=6)$} \\
\hline Job satisfaction & Job satisfaction survey & $30.00(0)$ & $30.00(0)$ \\
\hline Value of CST & Likert scale & - & $9.83(0.41)$ \\
\hline Satisfaction with CST & Likert scale & - & $9.83(0.41)$ \\
\hline
\end{tabular}

QoL-AD, Quality of Life in Alzheimer Disease Scale; MoCA, Montreal Cognitive Assessment; MARS-F, Memory Awareness Rating Scale-Functioning Subscale; MFD, memory functioning discrepancy. *Statistically significant difference between baseline and post-intervention scores.

disagree'. After the first CST session, CST facilitators rated each participant's level of engagement and level of confidence on Likert scales.

\section{CST intervention}

The CST intervention included 14 sessions, run for $~ 90$ minutes once/week for 14 weeks. Two CST facilitators at each site coordinated sessions using the CST manuals. For each group, a reality orientation board was displayed during sessions that included the group name, the date, and the location of the sessions. Sessions focussed on themes such as childhood, food, famous faces, current affairs, and word associations. Although weekly session themes were pre-determined by the CST manual, facilitators adapted activities and levels of difficulty according to their group's cognitive abilities, interests, and gender mix. Further details of the CST programme have been described elsewhere (Spector et al. 2003).

\section{Post-intervention assessments}

Each of the measures completed at baseline were administered again after the intervention was complete (Table 1). In addition, CST participants, carers, and CST facilitators each provided Likert ratings of the value of CST, and of their satisfaction with the CST programme.

\section{Qualitative interviews}

Post-intervention interviews were conducted by the primary researcher with four CST participants, six carers, and four CST facilitators. The interviews supplemented quantitative data by providing a personalised account of the impact of CST. A semi-structured interview design was incorporated that included four guiding questions: (1) Can you describe your overall experience and opinion of CST? (2) How satisfied were you with the programme? Explain. (3) Would you recommend CST to others? Why/why not? (4) Is there anything you would change? 


\section{Results}

\section{Baseline versus post-intervention scores}

The data met assumptions of normality and homogeneity of variances. Paired samples $t$-tests were conducted to analyse differences between means at baseline and post-intervention (Table 1). Results showed significant differences between baseline and post-intervention scores for CST participants self-rated satisfaction with cognitive performance $(t=-3.51, \mathrm{df}=$ $19, p=0.002)$, CST facilitators' ratings of participants' level of engagement $(t=-2.18, \mathrm{df}=15, p=0.046)$ and confidence $(t=-2.46, \quad \mathrm{df}=15, \quad p=0.026)$, and improvements on the MARS-F were marginally significant $(t=-2.04, \mathrm{df}=19, p=0.055)$. Positive MARS-F MFD scores at baseline $(\mathrm{M}=0.11$, s.D. $=0.68)$ and post-intervention $(\mathrm{M}=0.30$, S.D. $=0.57)$ indicated that participants rated their memory functioning more positively than their carers; with better agreement at baseline compared with post-intervention. Scores on the following outcomes of interest improved subsequent to CST (see Table 1), but did not reach statistical significance: MoCA, $p=0.572$; quality of life self-rated, $p=0.763$; cognitive ability, $p=0.152$; carer rated satisfaction with cognitive performance, $p=0.194$; well-being, $p=0.337$; and carer-rated engagement, $p=0.327$; and confidence, $p=0.723$; carers well-being, $p=0.115$. Scores on the informant QoL-AD measure were lower at follow-up compared with baseline, but the difference was not significant (quality of life carer-rated, mean difference $=0.25, p=0.874$ ).

\section{Correlational analysis}

\section{CST participants}

Pearson's correlation coefficient was used to determine whether relationships existed between scores for CST participants (Table 2). Baseline self-rating scores on the MARS-F showed moderate to strong positive correlations with participants' baseline quality of life (QoL-AD; $r=0.804, p<0.001)$, well-being $(r=0.609, p=0.004)$, satisfaction with cognitive performance $(r=0.518$, $p=0.019)$, and cognitive ability $(r=0.753, p<0.001)$; whereas post-intervention MARS-F scores showed moderate positive correlations with participants' post-intervention quality of life $(r=0.491, p=0.028)$, well-being $(r=0.509, p=0.022)$, and satisfaction with cognitive performance $(r=0.612, p=0.004)$.

Baseline self-rating scores for cognitive ability were strongly positively correlated with baseline scores for quality of life $(r=0.720, p<0.001)$, well-being $(r=0.674$, $p=0.001$ ), and satisfaction with cognitive performance $(r=0.682, p=0.001)$. Quality of life at baseline was positively correlated with baseline well-being $(r=0.638$, $p=0.002)$. Similarly, baseline scores for satisfaction with cognitive performance correlated with quality of life $(r=0.459, \quad p=0.042)$ and well-being $(r=0.606$, $p=0.005)$ at baseline, as well as post-intervention

Table 2. Pearson's correlations between scores for cognitive stimulation therapy participants that reached statistical significance

\begin{tabular}{|c|c|c|c|c|}
\hline & \multicolumn{4}{|c|}{ Baseline scores } \\
\hline & Quality of life & Well-being & Satisfaction cognitive performance & Cognitive ability \\
\hline \multicolumn{5}{|l|}{ Baseline scores } \\
\hline MARS-F & $r=0.804^{* *}$ & $r=0.609^{* *}$ & $r=0.518^{*}$ & $r=0.753^{* *}$ \\
\hline Cognitive ability & $r=0.720^{* *}$ & $r=0.674^{* *}$ & $r=0.682^{* *}$ & - \\
\hline Satisfaction cognitive performance & $r=0.459^{*}$ & $r=0.606^{* *}$ & - & - \\
\hline \multirow[t]{2}{*}{ Quality of life } & - & $r=0.638^{* *}$ & - & - \\
\hline & Level of engagement & & Satisfaction cognitive performance & \\
\hline \multicolumn{5}{|l|}{ Post-intervention scores } \\
\hline $\mathrm{MoCA}$ & $r=0.569^{*}$ & - & - & - \\
\hline Well-being & - & - & $r=0.451^{*}$ & - \\
\hline \multirow[t]{3}{*}{ Cognitive ability } & - & - & $r=0.523^{*}$ & - \\
\hline & \multicolumn{4}{|c|}{ Post-intervention scores } \\
\hline & Quality of life & Well-being & Satisfaction cognitive performance & Cognitive ability \\
\hline \multicolumn{5}{|l|}{ Post-intervention scores } \\
\hline MARS-F & $r=0.491^{*}$ & $r=0.509^{*}$ & $r=0.612^{* *}$ & - \\
\hline Satisfaction cognitive performance & - & $r=0.769^{* *}$ & - & $r=0.660^{* *}$ \\
\hline
\end{tabular}

MoCA, Montreal Cognitive Assessment; MARS-F, Memory Awareness Rating Scale-Functioning Subscale. ${ }^{* *},{ }^{*}$ Correlation is significant at the $0.01,0.05$ level, respectively. 
Table 3. Themes and sub-themes emerging from the analysis of interview data with participants with dementia, carers, and cognitive stimulation therapy (CST) facilitators

\begin{tabular}{lll}
\hline Themes & 1. Positive opinions about CST & 2. Direct Benefits of CST \\
\hline Sub-themes & $\begin{array}{ll}\text { 1.1. Positive attitudes towards the intervention } \\
\text { 1.2. Satisfaction with, and recommendations for CST }\end{array}$ & $\begin{array}{l}\text { 2.1. Increased confidence } \\
\text { 2.2. Cognitive benefits } \\
\text { 2.3. Increased enjoyment/improved mood }\end{array}$ \\
\hline
\end{tabular}

ratings of well-being $(r=0.451, p=0.046)$ and satisfaction with cognitive performance $(r=0.523, p=0.018)$. Finally, post-intervention MoCA scores were correlated with baseline level of engagement $(r=0.569, p=0.034)$; and satisfaction with cognitive performance was strongly correlated with post-intervention well-being ( $r=0.769$, $p<0.001)$ and cognitive ability $(r=0.660, p=0.002)$.

Carers and CST participants

Pearson's correlation coefficient was also used to determine whether relationships existed between CST participants' and carers' scores. Interestingly, carers baseline ratings of CST participants' quality of life was strongly positively correlated with participants' postintervention ratings of well-being $(r=0.677, p=0.003)$ and satisfaction with cognitive performance ( $r=0.609$, $p=0.009$ ). Carers' baseline ratings of their own well-being also positively correlated with participants' self-rated well-being at baseline $(r=0.504, p=0.039)$, and post-intervention MARS-F informant ratings $(r=0.486, p=0.048)$.

Results also showed a moderate negative correlations between carers' baseline ratings of satisfaction with loved one's cognitive performance and both MARS-F participant self-ratings $(r=-0.484, p=0.049)$ and participants' self-rated well-being ( $r=-0.582, p=0.014)$ at baseline. Similarly, carers' baseline $(r=-0.593, p=0.012)$ and post-intervention ( $r=-0.687, p=0.002)$ MARS-F informant scores were negatively correlated with participants' self-rated quality of life post-intervention.

\section{Qualitative interview data}

The transcribed interview data were organised and subdivided into relevant themes, guided by the findings of Spector et al. (2011). Across the three groups (CST participants $n=4$, carers $n=6$, and CST facilitators $n=4$ ) two main themes and five sub-themes emerged (Table 3).

\section{Theme 1: positive opinions about CST}

\section{Sub-theme 1.1: positive attitudes towards the intervention}

Positive attitudes towards CST were strongly expressed from all three groups. CST participants stated that the intervention was positive and they enjoyed taking part. Some discussed how they were unsure about being involved at the outset but that they were glad they continued to attend. 'At the start I didn't think it was for me, but at the end I was glad I kept going, I had to take back my original thoughts!' This was reiterated by carers. Many were relieved to have an appropriate service available for those in the earlier stages of dementia; and most expressed their admiration at the overall content. 'Very positive, somewhere to go as we had nowhere before'. 'A great experience'. 'I was amazed at all they got through ... The extra detail was fantastic; the facilitators went above and beyond'. 'We travelled from the midlands to Galway for each session. We found the journey challenging but the course was a great help'. Facilitators found CST 'excellent', 'important', wellsuited to their client groups; and were impressed by participants' adherence to the programme. All facilitators highlighted the demand for CST, particularly after people had completed the programme. 'We get people calling from everywhere, they are willing to travel miles'. 'I see the good that comes out of CST with everyone'.

Sub-theme 1.2: satisfaction with, and recommendations for CST

When asked about their satisfaction with the programme, all of those interviewed expressed satisfaction and said that they would recommend CST to others: 'It should be routinely offered to people with dementia' (F). 'Geriatricians need to recommend it at diagnosis' (C). 'I would definitely recommend it, I felt good after it. I was disappointed when it ended. I'd love to do it again' (P). Participants discussed how the programme either met or exceeded their expectations: 'I didn't know what expect at first but it lived up to all my expectations'. 'It was better than I expected'. When asked what they would change, respondents either reported that they wanted more sessions and better resources, or that they would not change anything. 'More groups are required; we need resources for follow-up CST sessions' (F). 'Well one of the tasks was a bit difficult, but I was happy with it, I wouldn't change anything' (P). 


\section{Theme 2: direct benefits of CST}

\section{Sub-theme 2.1: increased confidence}

The increase in confidence of CST participants was a strong recurring theme throughout the interview data, with all three groups commenting on confidence levels. 'I could see it in the rest of the people there too; they were all a lot more confident after a few weeks' (P). 'It was great for my confidence, I don't be giving out to myself anymore' (P). 'His confidence increased and meeting people in the same situation helped him a $\operatorname{lot}^{\prime}(\mathrm{C})$. 'It helped to give him confidence and maximise his ability' (C). 'Those that were anxious at week 1 gained so much confidence as the programme developed' (F). 'It was such a difference to see people at the end of CST, their confidence levels had totally increased' (F).

\section{Sub-theme 2.2: cognitive benefits}

Both CST participants and carers reported improvements in areas of cognitive function including memory, attention, and concentration. 'I found it great, it kept me focused in the right direction. It helped me to learn ways of ... improving my memory' (P). 'I still use the techniques and things I learned' $(\mathrm{P})$. 'It encouraged her to do things more and increased her attention' (C). 'I can't say it improved his memory 100\% but it did help his concentration to improve' (C). Facilitators comments were consistent with those above; some discussed specific examples of observing cognitive benefits: 'They asked for help filling out the forms at the beginning but at the end they said "we can do them ourselves"!' 'At the beginning they would take ages to think of names (ball activity) but at the end they were flying through it'.

\section{Sub-theme 2.3: increasing enjoyment/improved mood}

Throughout the interviews, the majority of carers and all facilitators discussed the participants' enjoyment of CST or how the programme appeared to improve their mood overall. 'One client was sullen, withdrawn and quiet at the start - she bounced in after 14 weeks, a changed person!' (F). Carers in particular reported noticeable changes in mood. 'He thought he wouldn't be able for it at first so he went in cranky but came out on top of the world' (C). 'After the sessions she came out to me a brighter, happier person' (C). 'Before he joined the CST sessions his mood was low, but he's so much better now' (C). These comments were supported by reports from CST participants, most of whom suggested feeling better after the intervention sessions. 'I don't get frustrated with myself anymore. When I forget something I just relax and then it comes back to me. I used to get very frustrated but I don't anymore'. 'I felt good after it. I'd love to do it again'.

\section{Discussion}

\section{Results summary and interpretation}

A 14 session, once weekly community-based CST intervention resulted in significant baseline to postintervention improvements in participants' subjective cognitive function and satisfaction with their own cognitive performance; and in facilitator ratings of participants' levels of engagement and confidence. CST marginally improved MoCA scores but improvements were not significant. Our findings support results from prior studies that reported cognitive benefits of CST (Spector et al. 2003; Onder et al. 2005; Coen et al. 2011; Aguirre et al. 2012; Woods et al. 2012). Previous research has reported significant improvements in quality of life from baseline to post-intervention (Aguirre et al. 2012) and in intervention versus control groups' (Spector et al. 2003; Coen et al. 2011; Woods et al. 2012). We showed that although CST benefitted participants' self-rated quality of life and well-being, baseline to postintervention improvements were not significant.

In the pre-post intervention design study by Aguirre et al. (2012), they reported that CST benefitted cognitive function (MMSE) and quality of life of participants with dementia. Similar to our study, they reported nonsignificant improvements on self-rated QoL-AD scores, and informant QoL-AD scores worsened post-intervention; but they found significant baseline to postintervention improvements on alternative measures of quality of life. Their findings suggest that a larger sample size and alternatives to the QoL-AD measure may be advantageous in observing significant pre-post intervention improvements in cognitive function and quality of life scores for CST participants.

In many prior studies (e.g. Spector et al. 2003; Coen et al. 2011; Woods et al. 2012), the benefits of CST were observed in intervention versus control group comparisons as opposed to pre-post intervention comparisons, as in the current study. The inclusion of a comparative control group in our research would have been preferable in order to more accurately determine the impact of the intervention on the primary outcomes of interest. Without a control group, it is difficult to determine participants' improvement relative to them receiving no intervention. Both Coen et al. (2011) and Spector et al. (2003), as in our study, reported only marginal (non-significant) improvements in CST participants' cognitive function from baseline to post-intervention, but importantly in their studies, control group scores typically deteriorated over time, as is commonly observed (Woods et al. 2012). Future studies of community-based CST should, where possible, include a wait-list control group to allow for direct comparisons and consistent conclusions to be made regarding the impact of the intervention. 
Our results are of particular interest in relation to those reported by Cove et al. (2014), who similar to our study, conducted once weekly sessions of CST as opposed to the typical twice weekly sessions. Unlike our results, however, Cove et al. (2014) found no evidence of any benefits of once weekly CST for people with dementia. Discrepant results may be explained by the different study designs; or due to differences in measures used across studies. Cove et al. only administered standardised measures of cognition, quality of life, and quality of relationships with carers; whereas our study incorporated a number of subjective measures such as ratings of confidence and cognitive ability. Cove et al. acknowledged that positive outcomes in unmeasured domains may have been missed in their study, and suggested that a wider range of outcome measures be used in future. Our research is the first therefore to show that once weekly CST sessions, a format often better suited to service delivery in the community, can benefit participants with dementia.

Positive outcomes on quantitative measures were supported by qualitative interview data that reported post-intervention improvements in CST participants' confidence, mood, concentration, attention, and memory. Spector et al. (2011) similarly found that CST participants and carers reported improvements in participant confidence and cognitive function (concentration, alertness, memory). Regarding confidence in particular, the benefit of CST was demonstrated by both quantitative and qualitative evidence in our study. This is important as cognitive impairment can be compounded by an associated decrease in confidence (Spector et al. 2011), which can further impede daily functioning of people with dementia. CST may alleviate this effect by increasing individuals' confidence in their own abilities, and promoting a more active and engaged lifestyle.

Results from the correlational analysis showed that participants' subjective cognitive function (MARS-F, cognitive ability, and satisfaction with cognitive performance) was associated with their quality of life, well-being, and satisfaction with their own cognitive performance (see Table 2). Prior research has shown that poorer cognitive function is related to lower levels of psychological well-being and quality of life (Hurt et al. 2010; Gates et al. 2014). Our findings, however, show that the person with dementia's self-rated or subjective cognitive abilities are related to well-being and quality of life. These improvements in participant's confidence in their own abilities, perhaps through improved self-concept or self-efficacy (Gates et al. 2014), may indicate a pathway to improved quality of life and well-being from a cognitive intervention. Future CST studies should further investigate the relationship between self-rated cognitive abilities and quality of life.
There was a significant relationship between carer and participant well-being, and between carer well-being and their ratings of participant cognitive function. These findings perhaps unsurprisingly show that carers' well-being may be impacted by participants' well-being, and vice versa. The results also show, however, that the well-being of carers may impact how they interpret the cognitive functioning of the person with dementia. Previous discussions have addressed similar findings (e.g. de Bettignies et al. 1990; Clare, et al. 2004) and indicate that carers who are experiencing lower levels of emotional well-being may underestimate their loved one's level of functioning. This suggests that in some cases, carers may not provide the most accurate account of participant capabilities; a point that researchers and practitioners should be aware of when evaluating the efficacy of cognitive interventions through informant report measures.

We found somewhat inconsistent associations between carer and participant scores. For example, higher participant-rated cognitive function and well-being was associated with lower carer satisfaction with participants' cognitive performance; and higher participant-rated quality of life was associated with lower carer-rated cognitive function of participants. Previous studies of cognitive rehabilitation interventions have reported similar inconsistencies (e.g. Clare et al. 2010; Kurz et al. 2012). One explanation for these findings may be that participants were less aware of their cognitive difficulties than carers. Evidence suggests that damage to the brain caused by dementia may result in lower awareness (Agnew \& Morris, 1998); and discrepancies between carer and participant ratings may indicate that the person with dementia has less awareness of their cognitive capacity (Clare et al. 2004). Lower participant awareness may therefore result in more positive outcome scores for participants but more negative scores from their carers (Clare et al. 2004). This explanation is supported by the discrepant scores reported on the MARS-F MFD that showed that participants rated their memory functioning more positively than their carers. As mentioned previously, however, inconsistencies may also be explained by carer emotional well-being; with lower levels of well-being associated with underestimated cognitive function of the person with dementia. Future CST studies should further examine relationships between CST participant and carer outcomes. Clinician ratings of participant awareness could be obtained, along with carer and participant-rated outcomes, to determine whether participant awareness and/or emotional well-being of carers result in inconsistent participant and carer outcome scores.

\section{Limitations and suggestions for future research}

Evaluating a community-based intervention across four sites had its inherent limitations. First, there were 
likely to be differences in how the CST intervention was implemented from site to site. Although this may be considered problematic, CST is designed to be delivered in a client-centred manner by adapting the intervention to the needs of the group, and was therefore unavoidable. Second, facilitators specifically requested that outcome measures be as quick and easy to complete as possible. This led the primary researcher to develop brief non-standardised Likert scales to assess many of the outcomes of interest (as identified by the ASI). Standardised measures are preferable due to the availability of reliability and validity data, and also to allow comparisons across studies. Future community-based CST evaluations could employ fewer, more carefully selected standardised measures; perhaps only quality of life, objective and subjective cognitive function, and confidence.

Staffing resource limitations meant that it was not possible to run integrity and inter-rater reliability (IRR) checks on data. Facilitators were, however, provided with guidelines on administering questionnaires, had dementia and CST training, and were largely unaware of the overall outcomes of interest of the evaluation. Nonetheless, future research should ensure that IRR data can be calculated for outcome measurement. Some facilitators also reported difficulty with the added workload of gathering outcome data as well as running the intervention. Future, better-resourced studies would ideally allocate two independent, blind researchers to administer assessments and run IRR checks. This would allow facilitators to concentrate on running the intervention groups only.

Finally, the nature of the study meant that sample sizes were small, some follow-up data were not provided, and it was not possible to include comparative controls. Therefore, although our findings support those of prior CST studies, the results should be interpreted with caution. Future community-based CST studies in Ireland would greatly benefit from increased resources that would allow for larger sample sizes and a wait-list control group.

\section{Implications/conclusions}

Overall, this study demonstrated that CST delivered across 14 weekly sessions in community-based group contexts in Ireland had a positive impact on the lives of people with dementia, their families, and on staff. In the United Kingdom, as far back as 2007, cognitive stimulation was made available by almost a third of community mental health teams for older adults (National Audit Office Report, 2007). Although we have no official data, it is widely recognised that evidence-based cognitively stimulating interventions are not routinely available to community dwelling people with dementia in Ireland; a point reiterated by our interview respondents. With the NDS in place since 2014, it is time for the Government to support the provision of identified priorities; namely timely intervention and integrated services for people with dementia. If economic figures can be compared with those in the United Kingdom, providing CST could save the Health Service Executive millions per year compared with the use of antipsychotic medication and premature admission into long-term care. With little alternative support currently available, CST, followed ideally by maintenance CST would be an excellent starting point.

\section{Acknowledgements}

The authors wish to thank the Alzheimer Society of Ireland, particularly Emer Begley and Miriam Enwright who contributed to making this research possible. This research has developed from work initiated by the first author when employed by the NEIL programme in Trinity College Institute of Neuroscience, Trinity College Dublin. The authors thank Dr Sabina Brennan, Prof. Brian Lawlor, and Prof. Ian Robertson for their support during that time.

\section{Conflicts of Interest}

The research was commissioned by the Alzheimer Society of Ireland.

\section{Financial Support}

The research was funded by a grant awarded to the Alzheimer Society of Ireland by the Scheme to Support National Organisations; Department of Environment, Community and Local Government.

\section{Ethical Standards}

The authors assert that all procedures contributing to this work comply with the ethical standards of the relevant national and institutional committee on human experimentation with the Helsinki Declaration of 1975 , as revised in 2008. The study protocol was approved by the institutional review board of the participating institution. Written informed consent was obtained from all participants.

\section{References}

Agnew SK, Morris RG (1998). The heterogeneity of anosognosia for memory impairment in Alzheimer's disease: a review of the literature and a proposed model. Aging and Mental Health 2, 7-19.

Aguirre E, Hoare Z, Streater A, Spector A, Woods B, Hoe J, Orrell M (2012). Cognitive stimulation therapy (CST) for people with dementia - who benefits most? International Journal of Geriatric Psychiatry 28, 284-290. 
Aguirre E, Spector A, Streater A, Hoe J, Woods B, Orrell M (2011). Making a Difference 2. Hawker Publications: UK.

Aguirre E, Woods RT, Spector A, Orrell M (2013). Cognitive stimulation for dementia: a systematic review of the evidence of effectiveness from randomised controlled trials. Ageing Research Reviews 12, 253-262.

Batsch N, Miller RV (2009). An assessment of needs for serving individuals diagnosed with Alzheimer's disease and related dementias in the early stages. Alzheimer Care Today 10, 140-155.

Cahill S, O'Shea E, Pierce M (2012). Creating Excellence in Dementia Care: A Research Review for Ireland's National Dementia Strategy. Living with Dementia Programme TCD and Irish Centre for Social Gerontology, NUI Galway: Dublin and Galway.

Clare L (2008). Cognitive Rehabilitation: A Modular Handbook. Psychology Press: Hove, UK.

Clare L, Linden DE, Woods RT, Whitaker R, Evans SJ, Parkinson CH, van Paasschen J, Nelis SM, Hoare Z, Yuen KS, Rugg MD (2010). Goal-oriented cognitive rehabilitation for people with early-stage Alzheimer disease: a single-blind randomized controlled trial of clinical efficacy. The American Journal of Geriatric Psychiatry 18, 928-939.

Clare L, Whitaker CJ, Nelis SM (2010). Appraisal of memory functioning and memory performance in healthy ageing and early-stage Alzheimer's disease. Aging, Neuropsychology, and Cognition 17, 462-491.

Clare L, Wilson BA, Carter G, Roth I, Hodges JR (2002). Assessing awareness in early-stage Alzheimer's disease: development and piloting of the Memory Awareness Rating Scale. Neuropsychological Rehabilitation 12, 341-362.

Clare L, Wilson BA, Carter G, Roth I, Hodges JR (2004). Awareness in early-stage Alzheimer's disease: relationship to outcome of cognitive rehabilitation. Journal of Clinical and Experimental Neuropsychology 26, 215-226.

Clare L, Woods RT, Moniz-Cook ED, Orrell M, Spector A (2003). Cognitive rehabilitation and cognitive training interventions targeting memory functioning in early stage Alzheimer's disease and vascular dementia. Cochrane Database for Systematic Reviews, article no. CD003260. doi:10.1002/14651858.CD003260.

Coen RF, Flynn B, Rigney E, O'Connor E, Fitzgerald L, Murray C, Dunleavy C, McDonald M, Delaney D, Merriman N, Edgeworth J (2011). Efficacy of a cognitive stimulation therapy programme for people with dementia. Irish Journal of Psychiatric Medicine 28, 145-147.

Cove J, Jacobi N, Donovan H, Orrell M, Stott J, Spector A (2014). Effectiveness of weekly cognitive stimulation therapy for people with dementia and the additional impact of enhancing cognitive stimulation therapy with a carer training program. Clinical Interventions in Aging $\mathbf{9}$, 2143-2150.

de Bettignies BH, Mahurin RK, Pirozzolo FJ (1990). Insight for impairment in independent living skills in Alzheimer's disease and multi-infarct dementia. Journal of Clinical and Experimental Neuropsychology 12, 355-363.

Department of Health (2014). The Irish National Dementia Strategy. Department of Health: Dublin (http:/ / health.gov.
ie/wp-content/uploads/2014/12/30115-National-DementiaStrategy-Eng.pdf). Accessed 22 August 2015.

Folstein MF, Folstein SE, McHugh PR (1975). 'Mini-mental state'. A practical method for grading the cognitive state of patients for the clinician. Journal of Psychiatric Research 12, 189-198.

Gates N, Valenzuela M, Sachdev PS, Fiatarone Singh MA (2014). Psychological well-being in individuals with mild cognitive impairment. Clinical Interventions in Aging 9, 779-792.

Grady CL, McIntosh AR, Beig S, Keightley ML, Burian H, Black SE (2003). Evidence from functional neuroimaging of a compensatory prefrontal network in Alzheimer's disease. Journal of Neuroscience 23, 986-993.

Health Information and Quality Authority (HIQA) (2008). National Quality Standards for Residential Care Settings for Older People in Ireland. Health Information and Quality Authority (https://www.hiqa.ie/system/files/ HIQA_Residential_Care_Standards_2008.pdf). Accessed 16 August 2015.

Hurt CS, Banerjee S, Tunnard C, Whitehead DL, Tsolaki M, Mecocci P, Kloszewska I, Soininen H, Vellas B, Lovestone S (2010). Insight, cognition and quality of life in Alzheimer's disease. Journal of Neurological Neurosurgery and Psychiatry 81, 331-336.

Knapp M, Thorgrimsen L, Patel A, Spector A, Hallam A, Woods B, Orrell M (2005). Cognitive stimulation therapy for people with dementia: cost effectiveness analysis. British Journal of Psychiatry 2, 574-580.

Kurz A, Thone-Otto A, Cramer B, Egert S, Frolich L, Gertz HJ, Kehl V, Wagenpfeil S, Werheid K (2012). CORDIAL: cognitive rehabilitation and cognitivebehavioral treatment for early dementia in Alzheimer disease: a multicenter, randomized, controlled trial. Alzheimer Disease and Associated Disorders 26, 246-253.

Logsdon RG, Gibbons LE, McCurry SM, Teri L (1999). Quality of life in Alzheimer's disease: patient and caregiver reports. Journal of Mental Health and Aging 5, 21-32.

Matrix Evidence (2011). An Economic Evaluation of Alternatives to Antipsychotic Drugs for Individuals Living with Dementia. NHS Institute for Innovation and Improvement: Coventry (http://www.institute.nhs.uk/images/Call_to_Action). Accessed 23 August 2015.

Nasreddine ZS, Phillips NA, Bedirian V, Charbonneau S, Whitehead V, Collin I, Cummings JL, Chertkow H (2005). The Montreal Cognitive Assessment, MoCA: a brief screening tool for mild cognitive impairment. Journal of the American Geriatrics Society 53, 695-699.

National Audit Office (2007). Improving Services and Supports for People with Dementia: Report by the Controller and Auditor General. The Stationary Office: London.

National Dementia Summit Report (2012). Getting on with Life: Our Action Plan for Living with Dementia. Alzheimer Society of Ireland: Dublin.

NICE-SCIE (2007). Dementia: Supporting People with Dementia and their Carers in Health and Social Care: Clinical Guideline 42. The British Psychological Society \& the Royal College of Psychiatrists: London.

Onder G, Zanetti O, Giacobini E, Frisoni GB, Bartorelli L, Carbone G, Lambertucci P, Silveri MC, Bernabei R (2005). 
Reality orientation therapy combined with cholinesterase inhibitors in Alzheimer's disease: randomised controlled trial. British Journal of Psychiatry 187, 450-455.

Orrell M, Aguirre E, Spector A, Hoare Z, Woods RT, Streater A, Donovan H, Hoe J, Knapp M, Whitaker C, Russell I (2014). Maintenance cognitive stimulation therapy for dementia: single-blind, multicentre, pragmatic randomised controlled trial. British Journal of Psychiatry 204, 454-461.

Perneczky R, Wagenpfeil S, Komossa K, Grimmer T, Diehl J, Kurz A (2006). Mapping scores onto stages: mini-mental state examination and clinical dementia rating. American Journal of Geriatric Psychiatry 14, 139-144.

Pierce M (2012). Post Diagnostic Services and Supports: User Preferences. Living with Dementia Programme Trinity College Dublin (http://dementia.ie/publications/ overview / publications-overview). Accessed 8 May 2015.

Prince M, Bryce R, Ferri C (2011). World Alzheimer Report: The Benefits of Early Diagnosis and Intervention. Alzheimer's Disease International: London (http:/ / www.alz.co.uk/ research/WorldAlzheimerReport2011.pdf). Accessed 12 September 2015.

Spector A, Gardner C, Orrell M (2011). The impact of cognitive stimulation therapy groups on people with dementia: views from participants, their carers and group facilitators. Aging and Mental Health 15, 945-949.

Spector A, Orrell M, Davies S, Woods RT (2001). Can reality orientation be rehabilitated? Development and piloting of an evidence based programme of cognition-based therapies for people with dementia. Neuropsychological Rehabilitation 11, 377-397.

Spector A, Thorgrimsen L, Woods B, Orrell M (2006). Making a Difference: An Evidence Based Group Programme to Offer Cognitive Stimulation Therapy (CST) to People with Dementia. Hawker Publications: London.

Spector A, Thorgrimsen L, Woods B, Royan L, Davies S, Butterworth M, Orrell M (2003). Efficacy of an evidencebased cognitive stimulation therapy programme for people with dementia: randomised controlled trial. British Journal of Psychiatry 183, 248-254.

Streater A, Spector A, Aguirre E, Hoe J, Hoare Z, Woods R, Russel I, Orrell M (2012). Maintenance cognitive stimulation therapy (CST) in practice: study protocol for a randomized controlled trial. Trials 13, 91-111.

Van Steenoven I, Aarsland D, Hurtig H, Chen-Plotkin A, Duda JE, Rick J, Chahine LM, Dahodwala N, Trojanowski JQ, Roalf DR, Moberg PJ, Weintraub D (2014). Conversion between mini-mental state examination, Montreal Cognitive Assessment, and dementia rating scale-2 scores in Parkinson's disease. Movement Disorders: Official Journal of the Movement Disorder Society 29, 1809-1815.

Woods B, Aguire E, Spector A, Orrell M (2012). Cognitive stimulation to improve cognitive functioning in people with dementia (review). Cochrane Database for Systematic Reviews 2, CD005562, doi: 10.1002/14651858.CD005562.pub2. 